\title{
Quick assessment tool for assurance of structural safety in the building process
}

\author{
Karel C. TERWEL \\ Lecturer and researcher \\ structural design \& safety \\ Delft University of \\ Technology \\ Delft, the Netherlands \\ k.c.terwel@tudelft.nl
}

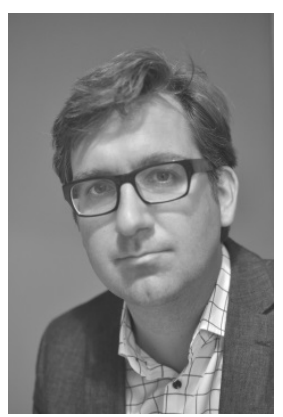

\author{
Sylvia J.T. JANSEN \\ Methodologist/ head of \\ fieldwork \\ Department OTB \\ Delft University of \\ Technology \\ Delft, the Netherlands \\ s.j.t.jansen@tudelft.nl
}

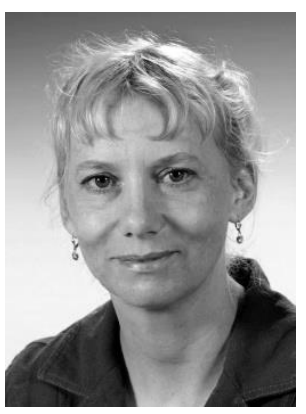

\section{Summary}

From forensic investigation it is known that many structural failures can be attributed to human errors and organizational factors. To provide project leaders with information on the current state of factors in the building process influencing structural safety, we developed a quick assessment tool. Logistic regression was used, based on data of influencing factors from a national questionnaire, to derive a function that predicts the probability of a successful outcome, regarding structural safety. The results show that a function with only the factors collaboration, risk analysis and control could predict a successful project correctly in $85 \%$ of cases, with collaboration as most determining factor. Although this method has limitations, it gives a quick indication of the degree in which problems regarding structural safety are to be expected. We believe that this tool has the potential to develop into a risk management tool.

Keywords: risk management, structural safety, building process, quality assurance

\section{Introduction}

Every building industry suffers from structural failures, sometimes resulting in the collapse of structures. Research shows that $70-90 \%$ of these failures is caused by human errors in the design and construction process $[1,2,3,4]$. Errors can occur in every working environment, although it is expected that the majority of employees intents to do a good job. It appears that organizational and human factors determine to a large extent if human errors are made [5].

For building industry it is important to know what human and organizational factors to what extent are influencing the successfulness of building projects regarding structural safety. When these aspects could be assessed during the building process, adequate measures can be taken to adjust the process and bring the project to success.

Within literature various lists of characteristics of the building process or the structure itself are given that might indicate the proneness to errors (e.g. [6]). However, usually the relative influence of various indicators is not quantified.

The goal of this study therefore is to design a quick assessment tool for managers which indicates during the design and construction process to what extent a successful project can be expected, regarding structural safety. In other words, this project aims to derive the relative influence of risk indicators, which are observable or measurable characteristics of the system or its constituents containing information about risk [7]. 


\section{Method}

\subsection{Possible indicators}

To design a safety assessment tool it should be known what the possible risk indicators are. Furthermore, quantification of these indicators is necessary.

For the possible indicators a list of human and organizational factors was set up, based on management literature and safety science [8].
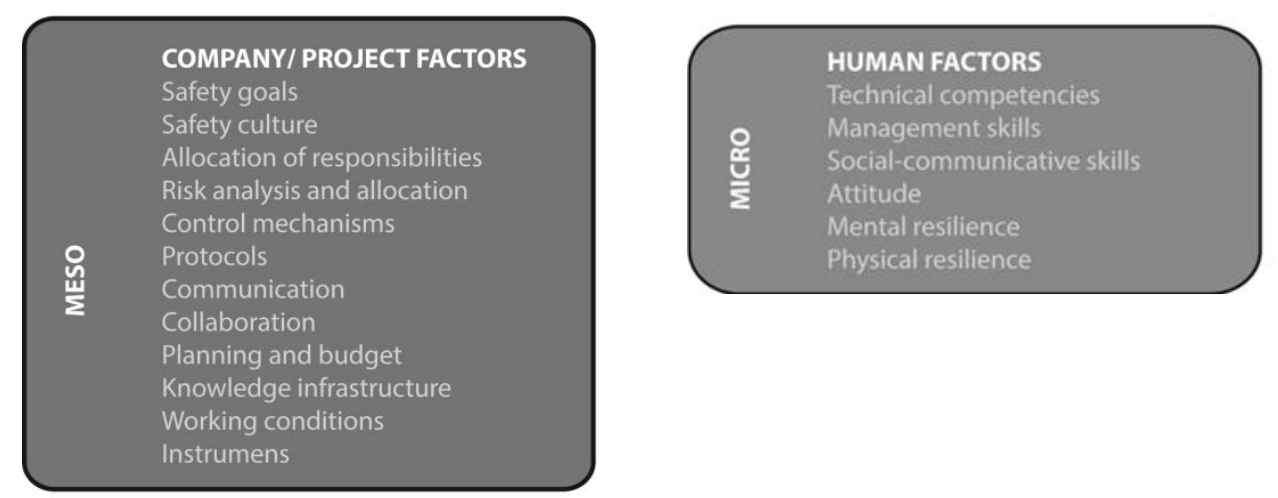

Figure 1: Possible company, project and human factors (based on [8])

These possible indicators include factors on project level (communication, control, allocation of responsibilities etc. between companies), on company level (same factors as project level but within the company) and human factors (like technical competencies, management skills and attitude, see figure 1). A suboptimal performance on each of these indicators could, theoretically, lead to an increased risk of structural failure. The list of indicators was tested in a pilot, which proved that the list was useable [9].

Subsequently, the importance of the resulting 39 indicators for structural safety was explored in a survey study with 236 respondents in the Netherlands [10]. We investigated which human and organizational factors determined the difference between a successful and less successful project regarding structural safety.

The predictors (39 possibly influencing factors) originally had five answering categories (totally disagree, partly disagree, neutral, partly agree, totally agree). However, we reduced this to three categories (disagree, neutral, agree) for two reasons. First, it simplifies the interpretation of the outcomes. Second, some categories on the 5-point Likert do not contain enough cases, which negatively influences the reliability of the statistical analyses.

In the current paper we will use the data of this national survey on structural safety to create a quick assessment tool for exploring the structural safety of a project. The method of logistic regression was used to derive a function that predicts the probability of a successful outcome of a project, based on just a small number of predictors. This method will be explained in more detail below.

\subsection{Logistic regression}

With logistic regression a bivariate outcome ( 0 or 1 ) is predicted on the basis of one of more predictors. The method allows us to predict which of two categories an object belongs to, given the presented information. In our study the two outcome categories are whether or not a project is termed less successful (0) or successful (1) by the respondent. The predictors are the scores on the 39 indicators for both a successful project and a less successful project, as explained above. 
The probability of an outcome can be calculated, according to Field [11] by logistic regression with:

(1) $\mathrm{p}(\mathrm{y})=\frac{1}{1+e^{-(b 0+b 1 * X 1+b 2 * X 2+\cdots+b n * X n)}}$

with:

$\mathrm{p}(\mathrm{y})=$ probability on the outcome of a variable

$\mathrm{b}_{0}=$ constant variable

$\mathrm{b}_{1}=$ weighting factor for predictor 1

$\mathrm{b}_{\mathrm{n}}=$ weighting factor for predictor $\mathrm{n}$

$\mathrm{X}_{1}=$ value for predictor 1

$\mathrm{X}_{\mathrm{n}}=$ value for predictor $\mathrm{n}$

The 39 statements are included as categorical predictors, the categories 'neutral' and 'agree' were compared against the 'disagree' category. We assume that a disagreement on one of the mentioned aspects has a negative effect on the successfulness of the project. Thus, it is expected that the absence of a certain aspect, such as a safety culture, is related to a lower probability of a successful project.

We are interested in a parsimoneous model, which means that the model only includes statistically significant predictors and that 'redundant' (in terms of statistical significance) predictors are omitted. In order to determine such a model, we used three different methods: "backward stepwise", "forward stepwise" and "backward elimination by hand". Both backward and forward are built-in algorithms in SPSS19 (as well as previous versions of SPSS). Briefly described, the backward algorithm starts with all potential predictors and eliminates predictors on statistical grounds, until all remaining predictors meet the demands of the minimum $\mathrm{p}$-value $(\mathrm{p}<0,05)$. The forward algorithm starts with a model with only the constant included and develops in a step-by-step way, by including only statistically significant predictors $(\mathrm{P}<0,05)$. Both methods are usually discouraged because inclusion or exclusion is based purely on statistical grounds and the researcher has no insight into this process. Backward elimination by hand starts with all potential predictors included in the model, as does automated backward elimination. Based on the results, the researcher decides which non-significant predictor is omitted (usually the one with the highest p-value) and the researcher looks into the results of the analysis without the particular variable. Then the process is repeated and the researcher again decides which non-significant predictor is omitted (if any), etc. This process enables the researcher to gain insight into the relationships between the various predictors and between the predictors and the dependent variable. As explained above, the use of stepwise models is usually regarded with suspicion, because unreliable results might be found due to a coincidental (idiosyncratic) combination of predictors in the specific sample. However, when the three different elimination methods result in about the same final model then reliability of the model can reasonably be established.

The logistic regression analysis yields odd ratios for each category of each predictor, except for the reference category. The odds ratio (OR) reflects the change in odds (which is the probability of the event occurring divided by the probability of the event not occurring) resulting from a unit change in the predictor (Field, 2009). The 95\% confidence interval (CI) around the odds ratio reflects its accuracy. The confidence interval indicates that for $95 \%$ of the time the true odds ratio in the population will fall within these limits (Field, 2009). Thus, if the confidence interval is large, then the result is less precise. In the current study we decided to eliminate a predictor from the analysis when the highest value in the $95 \%$ reliability interval was 20 times as high as the lowest value. For instance, if the odds ratio of a certain predictor is 14,9 with a $95 \%$ reliability interval between 1,4 and 153,9, this means that if one would draw 100 samples of the same size as the original sample and doing the same analysis, this would result in an odds ratio with a value between 1,4 and 153,9 
in 95 of these cases. A reason for such an unreliable result is the presence of only a few cases in one or more of the categories of a particular predictor.

In logistic regression, the fit of the model is represented by Nagelkerke's $r^{2}$, which is a pseudo measure for the amount of variance that is explained by the factors in the model. Nagelkerke's $\mathrm{r}^{2}$ is measured on a scale from 0 to 1 , with a higher value indicating a better fit.

\section{Results}

\subsection{Four models}

From the various methods of logistic regression, finally two groups of significant factors remain that give a reliable explanation of the outcomes. On the one hand these are risk analysis, collaboration and control and on the other hand: risk analysis, collaboration, knowledge infrastructure and technical skills. Apart from technical skills these are always factors on project level. Various combinations of the predictors of these two groups were used to set up four models.

These four different models are:

- Model 1: Collaboration

- Model 2: Risk analysis and collaboration

- Model 3: Risk analysis, collaboration and control

- Model 4: Risk analysis, collaboration, knowledge infrastructure and technical competencies

According to Terwel and Vambersky [8] collaboration can be defined as the way various project partners cooperate with each other. Risk analysis as the identification and assignment of risks associated with structural safety, of the building product and the building process. Control was defined as the way checking was performed (resulting in e.g. highlighting of design parts that were prone to mistakes during construction). Knowledge infrastructure was defined as a situation where technical and process knowledge of possible solutions are available (experience and knowledge are shared, learning from mistakes) and technical competencies are the demonstrated abilities to apply knowledge and skills for the design and construction of a structure.

The results of the logistic regression analysis are summarized in table 1 . The table clearly shows that with the inclusion of additional predictors, the individual influence of a particular predictor decreases (weight per predictor). However, the total explained variance of the model increases (Nagelkerke's $\mathrm{r}^{2}$ ).

The final rows of table 1 show the percentage of correct predictions according to the regression models. The reference for these models is the initial situation. In our study, a slightly higher number of successful projects $(n=247 ; 52 \%)$ than less successful project $(n=232)$ were included. This means that if all projects would have been termed successful, this would have been correct in 52\% of cases. Next, the probability of being a successful project is calculated on the basis of the predictors in the model and each case is classified into the category of a successful project or a less successful project, according to this probability. Finally, the predicted classification is compared to the observed classification (successful or not successful) in order to examine the value of the model with the predictors. 
Table 1: Results for various models

\begin{tabular}{|c|c|c|c|c|c|c|c|c|}
\hline & $\begin{array}{l}\text { Model } 1 \\
\text { Weight }\end{array}$ & $\begin{array}{l}\text { OR } \\
(95 \% \mathrm{CI}) \\
\end{array}$ & $\begin{array}{l}\text { Model } 2 \\
\text { Weight }\end{array}$ & $\begin{array}{l}\text { OR } \\
(95 \% \mathrm{CI})\end{array}$ & $\begin{array}{l}\text { Model } 3 \\
\text { Weight }\end{array}$ & $\begin{array}{l}\text { OR } \\
(95 \% \mathrm{CI}) \\
\end{array}$ & $\begin{array}{l}\text { Model } 4 \\
\text { Weight }\end{array}$ & $\begin{array}{l}\text { OR } \\
(95 \% \mathrm{CI})\end{array}$ \\
\hline Constant & $-1,90$ & & $-2,31$ & & $-2,68$ & & $-3,26$ & \\
\hline \multicolumn{9}{|l|}{ Collaboration } \\
\hline Disagree & --- & & --- & & --- & & --- & \\
\hline Neutral & 0,90 & $\begin{array}{l}2,47 \\
(1,23-4,96)\end{array}$ & 0,45 & $\begin{array}{l}1,57 \\
(0,75-3,31)\end{array}$ & 0,30 & $\begin{array}{l}1,34 \\
(0,62-2,91)\end{array}$ & 0,08 & $\begin{array}{l}1,09 \\
(0,46-2,54)\end{array}$ \\
\hline Agree & 3,04 & $\begin{array}{l}20,91 \\
(11,37-38,47)\end{array}$ & 2,30 & $\begin{array}{l}9,96 \\
(5,18-19,16))\end{array}$ & 1,89 & $\begin{array}{l}6,63 \\
(3,32-13,24)\end{array}$ & 1,70 & $\begin{array}{l}5,48 \\
(2,46-12,21)\end{array}$ \\
\hline \multicolumn{9}{|l|}{ Risk analysis } \\
\hline Disagree & & & --- & & --- & & --- & \\
\hline Neutral & & & 0,48 & $\begin{array}{l}1,62 \\
(0,82-3,18)\end{array}$ & 0,42 & $\begin{array}{l}1,52 \\
(0,76-3,07)\end{array}$ & 0,55 & $\begin{array}{l}1,73 \\
(0,80-3,74)\end{array}$ \\
\hline Agree & & & 1,59 & $\begin{array}{l}4,88 \\
(2,73-8,74)\end{array}$ & 1,28 & $\begin{array}{l}3,58 \\
(1,94-6,59)\end{array}$ & 1,40 & $\begin{array}{l}4,04 \\
(2,07-7,9)\end{array}$ \\
\hline \multicolumn{9}{|l|}{ Control } \\
\hline Disagree & & & & & --- & & & \\
\hline Neutral & & & & & 0,66 & $\begin{array}{l}1,94 \\
(0,92-4,10)\end{array}$ & & \\
\hline Agree & & & & & 1,18 & $\begin{array}{l}3,25 \\
(1,68-6,29)\end{array}$ & & \\
\hline \multicolumn{9}{|l|}{ Knowl. Infrast. } \\
\hline Disagree & & & & & & & --- & \\
\hline Neutral & & & & & & & 0,93 & $\begin{array}{l}2,53 \\
(1,16-5,54)\end{array}$ \\
\hline Agree & & & & & & & 1,13 & $\begin{array}{l}3,11 \\
(1,45-6,65)\end{array}$ \\
\hline \multicolumn{9}{|l|}{$\begin{array}{l}\text { Technical } \\
\text { competencies }\end{array}$} \\
\hline Disagree & & & & & & & --- & \\
\hline Neutral & & & & & & & $-0,02$ & $\begin{array}{l}0,98 \\
(0,34-2,83)\end{array}$ \\
\hline Agree & & & & & & & 0,78 & $\begin{array}{l}2,18 \\
(0,92-5,19)\end{array}$ \\
\hline Nagelkerke's r ${ }^{2}$ & 0,39 & & 0,44 & & 0,46 & & 0,50 & \\
\hline $\begin{array}{l}\text { Correct pred. of } \\
\text { successf. Proj.(\%) }\end{array}$ & $83,1 \%$ & & $77,3 \%$ & & $85,2 \%$ & & $85,3 \%$ & \\
\hline $\begin{array}{l}\text { Correct pred. of } \\
\text { less successf. } \\
\text { Proj. (\%) }\end{array}$ & $72,4 \%$ & & $78,9 \%$ & & $74,0 \%$ & & $75,3 \%$ & \\
\hline
\end{tabular}

\subsection{From four models to one}

For our quick assessment tool one model had to be selected. It was chosen to select those models with an optimum in the number of predictors and the correct prediction of the outcome.

Based on the various methods of logistic regression, four models were set up. Model 1 is the basic model which only includes the predictor collaboration. It gives a reasonable prediction for successful and less successful projects (see the lower row of Table 2).

To improve the predictive power it is possible to include extra predictors in the model. Model 2 additionally includes the variable risk analysis. The predictive values of less successful project improves on the detriment of the predictive value of successful projects.

Model 3 also includes the factor control. The likelihood of a right estimation of a successful and less successful model is increased compared to model 1 , and so is Nagelkerke's $r^{2}$. 
The increase in the successful prediction is very limited for model 4 when the factor control is substituted by knowledge infrastructure and technical skills. When a model with risk analysis, collaboration, control, knowledge infrastructure and technical competencies is composed, technical competencies and control are not significant anymore.

Hence, it can be concluded that model 3 gives a good prediction with a relatively low number of variables, thus our goal of obtaining a parsimonious model was reached. Model 3 is used for our quick assessment tool to estimate the successfulness of a project.

Estimation of the successfulness of a project

In the data of the survey the ratio of successful and less successful projects is $48,6 \%: 51,4 \%$. In reality this ratio might be different.

The likelihood of a successful project can be calculated by logistic regression with formula 1 . The constant $b_{0}$ and the coefficients $b_{1}$ to $b_{n}$ in formula 1 are derived with regression analysis and were presented in table 1.

The regression-function from model 3 can be presented as:

(2) $\mathrm{p}(\mathrm{y})=\frac{1}{1+e^{-(-2,679+0,422 * X 1 n+1,275 * X 1 p+0,662 * X 2 n+1,18 * X 2 p+0,295 * X 3 n+1,892 * X 3 p)}}$

With:

$\mathrm{p}(\mathrm{y})=$ probability of a successful project regarding structural safety

$\mathrm{X} 1 \mathrm{n}=$ neutral assessment of the factor risk analysis

$\mathrm{X} 1 \mathrm{p}=$ positive assessment of the factor risk analysis

$\mathrm{X} 2 \mathrm{n}=$ neutral assessment of the factor control

$\mathrm{X} 2 \mathrm{p}=$ positive assessment of the factor control

$\mathrm{X} 3 \mathrm{n}=$ neutral assessment of the factor collaboration

$\mathrm{X} 3 \mathrm{p}=$ positive assessment of the factor collaboration

$\mathrm{X} 1$ to X3 have a dichotomous value 0 (not given) or 1 (given)

The neutral and positive assessments are always compared against the negative assessment (the reference). It can be noticed that the coefficient for the positive assessment of the factor collaboration is the highest in this model. Collaboration therefore is of major influence within the function.

The results show that a function with only the factors collaboration, risk analysis and control can predict a successful project correctly in $85 \%$ of cases and a less successful project correctly in $74 \%$ of cases (see table 1). Furthermore, using the assessment values in the formula shows that a project that scores negatively on the three factors has a probability of only $6 \%$ of being a successful project whereas a project that scores positively on all three factors has a probability of $84 \%$.

Based on the regression-function, table 2 presents the likelihood of a successful project regarding structural safety for various assessment options (disagree, neutral, agree) for the three determining factors

Every cell of the table is a combination of the assessment of the three predictors. The first number is for risk analysis, the second number for control and the third number for collaboration. A ' 1 ' reflects a negative assessment of the predictor, a ' 2 ' reflects a neutral assessment and a ' 3 ' a positive assessment. A combination '123' for instance reflects a negative assessment of risk analysis, a neutral assessment of control and a positive assessment of collaboration. The colour 
(grey scale) depicts the likelihood of a successful project. The lighter the colour, the higher the probability of a successful project.

Table 2 Likelihood of successful project

\begin{tabular}{|c|c|c|c|c|c|c|c|c|}
\hline 111 & 112 & 113 & 121 & 122 & 123 & 131 & 132 & 133 \\
\hline 211 & 212 & 213 & 221 & 222 & 223 & 231 & 232 & 233 \\
\hline 311 & 312 & 313 & 321 & 322 & 323 & 331 & 332 & 333 \\
\hline
\end{tabular}

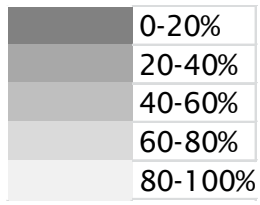

From table 2 it can be concluded again, that the factor collaboration is determining for the outcomes. If there is no good collaboration (the third number is smaller than 3 ), the probability of a successful project is smaller than $40 \%$. If the collaboration is regarded as good, the likelihood of a successful project will be over $60 \%$, regardless the assessment of the other factors.

The method gives a quick indication of the degree in which problems regarding structural safety are to be expected. It is easy to use the derived formula; application in an app is possible. Clients, contractors or structural engineers can easily assess their projects on collaboration, risk analysis and control and an indication is provided to what extent the project is vulnerable to structural deficiencies.

\section{Discussion}

Our study has shown the development of a quick assessment tool for exploring structural safety, which was based on the responses to 39 potential indicators of structural safety by 236 respondents. We concluded that a tool based on the perception with regard to collaboration, risk analysis and control would be sufficient to predict successful projects in $85 \%$ of cases and less successful projects in $74 \%$ of cases. However, our research has some limitations.

A first remark on the used methodology is that for logistic regression one of the requirements is that cases are independent. However, in our survey every respondents was asked to assess a successful and a less successful project on 39 aspects. Thus, the responses were related. The advantage of such an approach is that the influence of personal characteristics is decreased because one and the same person evaluates both a successful and a less successful project at the same time. For example, if a respondent is vulnerable for the tendency to provide high ratings, this might have occurred to a similar extent for both the successful and the less successful project. The drawback of related responses is that there might be a strong correlation in responses between cases that belong to a particular group, which is incorrectly considered to be an effect of the analysis but is in fact a group effect. However, this problem probably does not affect our analyses because we have only two projects (no more) for almost each respondent.

Second, not all variance is explained by the formula. Nagelkerke's $r^{2}$, which is a pseudo measure for the amount of explained variance, is 0,464 for the derived model, while it might be in an ideal situation 1,0 . This means that more than $50 \%$ of the variance in the outcome is not explained by our factors but by other predictors. For example, this formula does not include project characteristics, like type or size of the project, which might influence the relevance of various factors.

In addition, because some answering categories were hardly used by the respondents we had to combine categories. The "moderately agree” and “completely agree” categories were pooled as well 
as the "moderately disagree" and "completely disagree" categories. This reduces refinement of the predictions.

Finally, the predictors in the survey (presence of control, quality of collaboration, risk analysis) are broadly defined and interpretation will depend on subjective perception. It is recommended to refine the categories of the predictors and to apply measurable control variables (like the percentage of time devoted to control, the number of conflicts per week etc). In this way the confidence interval for the predictors might be reduced.

\section{Conclusions}

In this paper a tool is explained which estimates the probability of a successful project, regarding structural safety, based on a limited number of predictors, which were derived on the experience of past projects. Although the tool is based on data from a Dutch questionnaire, it is expected that it can be relevant for various countries.

It was concluded that a model with an assessment of collaboration, risk analysis and control correctly predicts a successful project regarding structural safety in $85 \%$ of the cases. The likelihood of a successful project is $84 \%$ when all factors were assessed positively. This indicates that attention for these factors in current building projects deserves attention from project developers, project managers, structural engineers and contractors. The tool is based on data from a Dutch questionnaire, but can be relevant for various countries.

Although, the used method has some limitations, this tool has the potential to develop into a useful risk management tool. In future studies, data on project characteristics can be included and more cases can be collected. By constantly updating the logistic regression function with new data, the model will get learning capacity.

In addition, it is possible to develop a certification system, like BREEAM, where clients can get an impression of the quality of the product based on an assessment of process and product parameters. This might give added value to a project, just as is the case with projects with high BREEAM scores for sustainable performance. To develop a certification system, data sources of failure databases, surveys and additional expert judgement are necessary to evaluate the various factors.

\section{References}

[1] SCHNEIDER J. and MATOUSEK M., Untersuchungen zur Struktur des Sicherheitsproblems bei Bauwerken. Zürich Institut für Baustatik und Konstruktion, ETHZ, 1976.

[2] ELLINGWOOD B. E., "Design and construction error effects on structural reliability." Journal of structural engineering 13(2): 409-422, 1987.

[3] FRUEHWALD E., SERRANO E., et al., Design of safe timber structures- how can we learn from structural failures in concrete, steel and timber? Lund, Lund Institute of Technology, 2007.

[4] TERWEL, K. C., BOOT W.F., NELISSE, R.M.L., "Structural unsafety revealed by failure databases." Forensic Engineering (FE1): 16-26, 2014.

[5] DEKKER, S., The field guide to understanding human error. Aldershot, Ashgate publishing limited, 2006.

[6] PUGSLEY A., The safety of structures. London, Edward Arnold, 1966.

[7] FABER M.H. et al., (2008). Risk Assessment in Engineering: Principles, System Representation and Risk Criteria, Joint Committee on Structural Safety.

[8] TERWEL, K. C. and VAMBSERKY J.N.J.A., Possible Critical Structural Safety Factors: a literature review.

Forensic engineering 2012: Gateway to a safer tomorrow. A. M. Dolhon et al. San Francisco, ASCE, 2012.

[9] DIJKSHOORN G. W., TERWEL K.C., GULDENMUNDT F., Determining critical factors to avoid failures in the building process. Safety, Reliability and Risk analysis: Beyond the horizon - ESREL 2013. R. D. J. M. Steenbergen et al. Amsterdam, Taylor \& Francis Group, 2014.

[10] TERWEL, K. C. and JANSEN S. J. T. "Critical factors for structural safety in the design and construction phase." Journal of performance of constructed facilities, ASCE, accepted for publication.

[11] FIELD A., Discovering statistics using SPSS. London, Sage, 2005. 